\title{
I Kritische Analyse des Einflusses eines Migrationshintergrundes auf das Anlageverhalten in ausgewählten Kategorien
}

\author{
Philippe Krahnhof ${ }^{\star}$,Eric Frère ${ }^{\star *}$ und Alexander Zureck ${ }^{\star * *}$
}

\section{Zusammenfassung}

Der vorliegende wissenschaftliche Beitrag analysiert das Anlageverhalten von Personen mit ${ }^{2}$ und ohne Migrationshintergrund in Deutschland. Es werden Gemeinsamkeiten sowie Unterschiede in den Kategorien Beratung in finanziellen Angelegenheiten, Finanzprodukte, Anlageziele sowie Wertentwicklung untersucht. Die Auswertung basiert auf einem Datensatz vom isf Institute for Strategic Finance an der FOM Hochschule für Oekonomie \& Management in Essen aus dem Jahre 2019. Der standardisierte Fragebogen umfasste rund 36 Fragen. Bei der Onlinebefragung haben Personen im Alter von 18 bis 56 Jahren teilgenommen. Zur Auswertung wurden vor allem deskriptive statistische Verfahren herangezogen. Die Studie kann einerseits die Forschungsergebnisse aus dem vergangenen Jahr untermauern, andererseits konnten in der Rubrik Finanzprodukte neue Erkenntnisse gewonnen werden. Während bei der Rubrik Beratung in finanziellen Angelegenheiten starke Übereinstimmungen zu verzeichnen sind, unterscheiden sich vor allem die Anlageziele sowie die selektierten Assets zwischen Personen in Deutschland mit und ohne Migrationshintergrund im Betrachtungszeitraum 2019.

Schlagwörter: Migrationshintergrund, Anlageverhalten, Wertentwicklung, Finanzberatung

Critical analysis of the influence of a migration background on investment behavior in selected categorie

Abstract

This scientific article analyzes the investment behavior of people with and without a migration background in Germany. Similarities and differences in the categories "Advice on financial matters, financial products, investment goals and performance" are examined. The evaluation is based on a dataset from the isf Institute for Strategic Finance at the FOM University of Economics \& Management in Essen from 2019. The standardized questionnaire comprised around 36 questions. People between the ages of 18 and 56 took part in the online survey. Above all, descriptive statistical methods were used for the evaluation. On the one hand, the study can underpin the research results from last year; on the other hand, new findings were gained in the "Financial Products" section. While there are strong similarities in the section "Advice on financial matters", the investment goals and the selected assets differ between people in Germany with and without a migration background in the 2019 observation period.

Keywords: migration background, investment behavior, performance, financial advice

\footnotetext{
IIIIIIIIIIIIIIIIIIIIIIIIIIIIIIIIIII

* Philippe Krahnhof, isf Institute for Strategic Finance, FOM Hochschule für Oekonomie \& Management, Essen E-Mail:philippe. krahnhof@fom-net.de.

** Eric Frère, isf Institute for Strategic Finance, FOM Hochschule für Oekonomie \& Management, Essen. E-Mail:eric.frere@ fom-isf.de

*** Alexander Zureck,isf Institute for Strategic Finance, FOM Hochschule für Oekonomie \& Management, Essen. E-Mail: alexander.zureck@fom-isf.de

Das Paper ist co-finanziert durch die Europäische Union im Programm Erasmus+ im Projekt (FKZ: 2019-1-CZo1-KA203-0613579). Das Projekt läuft noch bis zum 31.08.2021.

${ }^{1}$ Migrationshintergrund: Mindestens ein Elternteil stammt ursprünglich nicht aus Deutschland.
} 


\section{Einleitung}

Die Anzahl der deutschen Privatanleger und -anlegerinnen stieg im Betrachtungszeitraum von 2014 bis 2018 um rund 410.00o, dies entspricht einem prozentualen Zuwachs von etwa 9,9 Prozent (Deutsches Aktieninstitut 2019). Dieser Aspekt kommt Banken zugute, da sich diese aktuell in einem starken Veränderungsprozess befinden. Einerseits sinken die Zinserträge durch die anhaltende Niedrigzinsphase und kontinuierlich ansteigenden regulatorischen Anforderungen (Krahnhof/Zureck 2018; Zureck/Krahnhof 2019). Andererseits ist der Wettbewerbsdruck aufgrund von vielen neuen Marktteilnehmern im FinTech-Bereich gestiegen (Zureck/Krahnhof 2017). Somit kann die gezielte Kundenansprache von Privatanlegern und -anlegerinnen einen Bestandteil von erfolgreichem Banking darstellen.

In diesem Kontext ist zu betonen, dass mehr als 20 Prozent der deutschen Bevölkerung einen Migrationshintergrund haben, wie Daten des statistischen Bundesamtes $\mathrm{zu}$ entnehmen ist. 2016 belief sich die absolute Zahl auf rund 18,6 Millionen Menschen. Diese Zahl stieg binnen drei Jahren auf 20,8 Millionen, sprich um rund 11,8 Prozent, an (Statistisches Bundesamt 2019). Mit Blick auf den anhaltenden Fachkräftemangel wird sich die Anzahl an Migranten und Migrantinnen in Deutschland in den kommenden Jahren weiter erhöhen (Mayer 2015). Demzufolge hat dieser Beitrag sowohl einen praktischen Mehrwert für den Bankvertrieb als auch für soziographisch politische Entscheidungen.

Dieser Artikel analysiert, ob sich das Anlageverhalten von Migranten und Migrantinnen gegenüber jenem von Nicht-Migranten und Nicht-Migrantinnen unterscheidet. Hierbei ist zu unterstreichen, dass Auswirkungen der verschiedenen Herkunftsländer nicht analysiert werden. Folglich kann diese empirische Ausarbeitung weitere Erkenntnisse zum Stand der Financial-Literacy-Forschung im deutschsprachigen Raum liefern.

\section{Stand der Forschung}

Die Vielzahl aktueller wissenschaftlicher Beiträge stammt aus den Vereinigten Staaten von Amerika (de Bassa Scheresberg 2013; Haliassos et al. 2017; Lusardi/ Mitchell 2011; Lusardi et al. 2010), jedoch existieren auch Studien zum europäischen Raum. Europäische Studien zeigen, dass kulturelle Unterschiede bei Geld- anlagen zwischen Migranten und Migrantinnen und Einheimischen vor allem auf unterschiedliche Bildungsstände zurückzuführen sind. Im Allgemeinen beeinflussen kulturelle, soziale und politische Unterschiede die Finanzkompetenz (Zureck/Svoboda 2015).

Ohnehin nimmt das Thema Financial Literacy einen wesentlichen Stellenwert in der heutigen Zeit ein (DIA Vorsorge 2019). In der Literatur existieren unterschiedliche Begriffsdefinitionen zu Financial Literacy. Während „Financial“ als generelles Finanzwesen verstanden werden kann, wird „Literacy“ nicht einheitlich definiert (Schneider-Reißig 2018). In dieser wissenschaftlichen Arbeit wird Financial Literacy als Finanzkompetenz bzw. als Fähigkeit, mit privaten und persönlichen Finanzthemen eigenständig umgehen zu können, verstanden. Folglich umfasst der Begriff die Expertise über Finanzprodukte und -konzepte, mathematische Fähigkeiten sowie Kenntnisse über die Durchführung von Budgetplanungen (Hasting et al. 2013). Ausgeprägte Financial Literacy ist demnach eine Möglichkeit für Individuen, souverän mit der Liberalisierung des Finanzsystems, und hier besonders des Rentensystems, umzugehen. Positive Financial Literacy kann sich beispielsweise darin äußern, dass verstärkt in die private Altersvorsorge investiert und somit dem Wohlfahrtsverlust proaktiv entgegengewirkt wird. Somit kann Financial Literacy als Bestandteil der stückweisen Abkopplung des Individuums vom wohlfahrtsstaatlichen System verstanden werden. Durch diese Abkoppelung ermöglicht positive Financial Literacy unter anderem die Erzielung höherer Renditen (Hung et al. 2009; Ferguson 2002). Somit lassen sich die Financial-Literacy-Gedankengänge dem Neoliberalismus zuordnen (Arthur 2012).

Trotz der Relevanz dieses Themengebietes spielt die Finanzbildung in deutschen Schulen eine untergeordnete Rolle (Deutsches Aktieninstitut 2019). So ist einer Studie von ING aus dem Jahr 2017 beispielsweise zu entnehmen, dass rund 50 Prozent der jungen Deutschen ihre persönliche Finanzkompetenz als unzureichend bezeichnen. Folglich belegt Deutschland in internationalen Studien überwiegend hintere Ränge (Köhler 2017). Diese empirischen Befunde werden zusätzlich durch eine Studie der Organisation for Economic Co-operation and Development (OECD) aus dem Jahr 2005 unterstrichen, die auf mangelhafte Finanzbildung in den OECD-Staaten hinweist (Financial Literacy \& Education Russian Trust Fund).

Die Ausprägung von Financial Literacy weist in einigen europäischen Ländern große Unterschiede auf. 
Beispielsweise ist die finanzielle Kompetenz im nördlichen Italien höher als im Süden des Landes. Gleichwohl ist Financial Literacy nicht in allen nördlichen Regionen in hohem Maße vorhanden (Haliassos et al. 2017; Lusardi/Mitchell 2011). Zusätzlich sind Unterschiede zwischen städtischen und ländlichen europäischen Gebieten zu erkennen. So ist beispielsweise die Finanzbildung in städtischen Regionen Russlands stärker ausgeprägt als in ländlichen Regionen (Haliassos et al. 2017). Während in Deutschland das Thema in den vergangenen Jahren weniger stark forciert wurde, sind in den USA entsprechende Bildungsangebote im Alltag deutlich präsenter. So wird dort u. a. in den Printmedien oder sogar in Supermärkten verstärkt auf Bildungsmaßnahmen hingewiesen (YourHub 2019).

Während in den vergangenen Jahrzehnten die gesetzlichen Rentenleistungen für die Mehrheit der deutschen Bevölkerung ausreichend waren, müssen sich heute immer mehr Menschen mit der privaten Altersvorsorge auseinandersetzen (Zureck et al. 2018). Das ist ein Grund, warum die Deutschen normalerweise sichere oder eher renditearme Anlagen präferieren. Dieses Verhalten ist risikoavers und führt dazu, dass sie auf lange Sicht finanzielle Nachteile erleiden, da Aktienrenditen oder andere risikoreichere Investments mit einer die Inflationsrate übersteigenden Verzinsung fehlen (Lusardi/Mitchell 2011). Dieses Phänomen wird auch als Bestandteil der finanziellen Repression charakterisiert (Homburg et al. 2013). Die Literatur definiert die finanzielle Repression als staatliche Strategie, die Staatsverschuldung mittels verschiedener Maßnahmen, Gesetze sowie Vorschriften abzubauen (Belke/ Keil 2013). Der Begriff wurde in den 1970er-Jahren unter anderem von den Autoren Shaw (1973) sowie McKinnon (1973) geprägt. Das beispielsweise in Europa herrschende Niedrigzinsumfeld, beeinflusst durch den Leitzins der Europäischen Zentralbank (EZB), ist ein staatliches Mittel im Rahmen der strategischen finanziellen Repression (Belke/Keil 2013). Somit kommt der individuellen Abkoppelung von staatlichen Wohlfahrtssysteme insbesondere mit Blick auf das aktuelle Zinsniveau und die europäischen Staatsverschuldungen (Institut der Wirtschaftsprüfer 2019) heutzutage ein signifikanter Stellenwert zu (Zimmermann/Baier 2012).

Es besteht ein Zusammenhang zwischen der Financial Repression und der Financial Literacy. Je höher die Financial Literacy des Individuums ausgeprägt ist, desto eher werden Maßnahmen zur privaten Vorsorge ergriffen und infolgedessen den Folgen der finanziellen Repression entgegengewirkt (Vargas 2012).
Das skizzierte zurückhaltende Investitionsverhalten der deutschen Bevölkerung, kombiniert mit der zu erwartenden Niedrigzinsphase, ist unter anderem ein Aspekt, warum Studien eine steigende Altersarmut prognostizieren. Insbesondere Langzeitarbeitslose, aber auch Menschen mit Migrationshintergrund, die nicht ihr gesamtes Berufsleben in Deutschland verbrachten, sind von der Altersarmut gefährdet. Da in den meisten Herkunftsländern der angesprochenen Migranten und Migrantinnen kein Rentensystem existiert, wird u. a. das Risiko der Altersarmut verdeutlicht (Haan et al. 2017).

Folglich dient diese wissenschaftliche Arbeit dazu, neue Erkenntnisse durch den gesammelten Datensatz im Kontext des Themas Financial Literacy zu gewinnen.

\section{Empirisches Vorgehen}

Die zugrunde liegende empirische Untersuchung basiert auf einer quantitativen Datenerhebung des isf Institute for Strategic Finance an der FOM Hochschule für Oekonomie \& Management in Essen aus dem Jahr 2019. Der standardisierte Fragebogen umfasste 36 Fragen, bestehend aus geschlossenen und offenen Fragestellungen. Bei der Onlinebefragung haben sowohl Externe als auch Lehrende sowie Studierende der FOM Hochschule im Alter zwischen 18 und 56 Jahren teilgenommen. Hierbei ist jedoch zu unterstreichen, dass sich die Mehrheit der erhaltenen Antworten auf die Altersspanne zwischen 18 und 30 Jahren bezieht. Folglich handelt es sich um einen selektiven Befragungskreis. Das Nettohaushaltseinkommen der Befragten lag überdurchschnittlich hoch bei 3.800,00 Euro.

Um Rückschlüsse auf die Kategorien finanzielle Angelegenheiten, Finanzprodukte, Anlageziele der Befragten und Wertentwicklung der getätigten Investitionen zu bekommen, wurden einzelne Fragen der Onlinebefragung herangezogen und analysiert. Hierbei ist zu unterstreichen, dass die einzelnen Fragen unabhängig voneinander ausgewertet wurden. Folglich kann es zu inhaltlichen Überschneidungen zwischen den einzelnen Fragestellungen kommen. Zur Auswertung der erhaltenen Fragen wurden Verfahren der deskriptiven Statistik herangezogen.

Während 2019 13,86 Prozent der Befragten einen Migrationshintergrund aufwiesen, waren dies 2018 15,2 Prozent (Özcan et al. 2018; Zureck 2018). Bei der Frage bezüglich der Einschätzung des generellen Verständnisses von Finanzthemen schätzen 62,75 Prozent der Befragten mit Migrationshintergrund ihr subjektives 


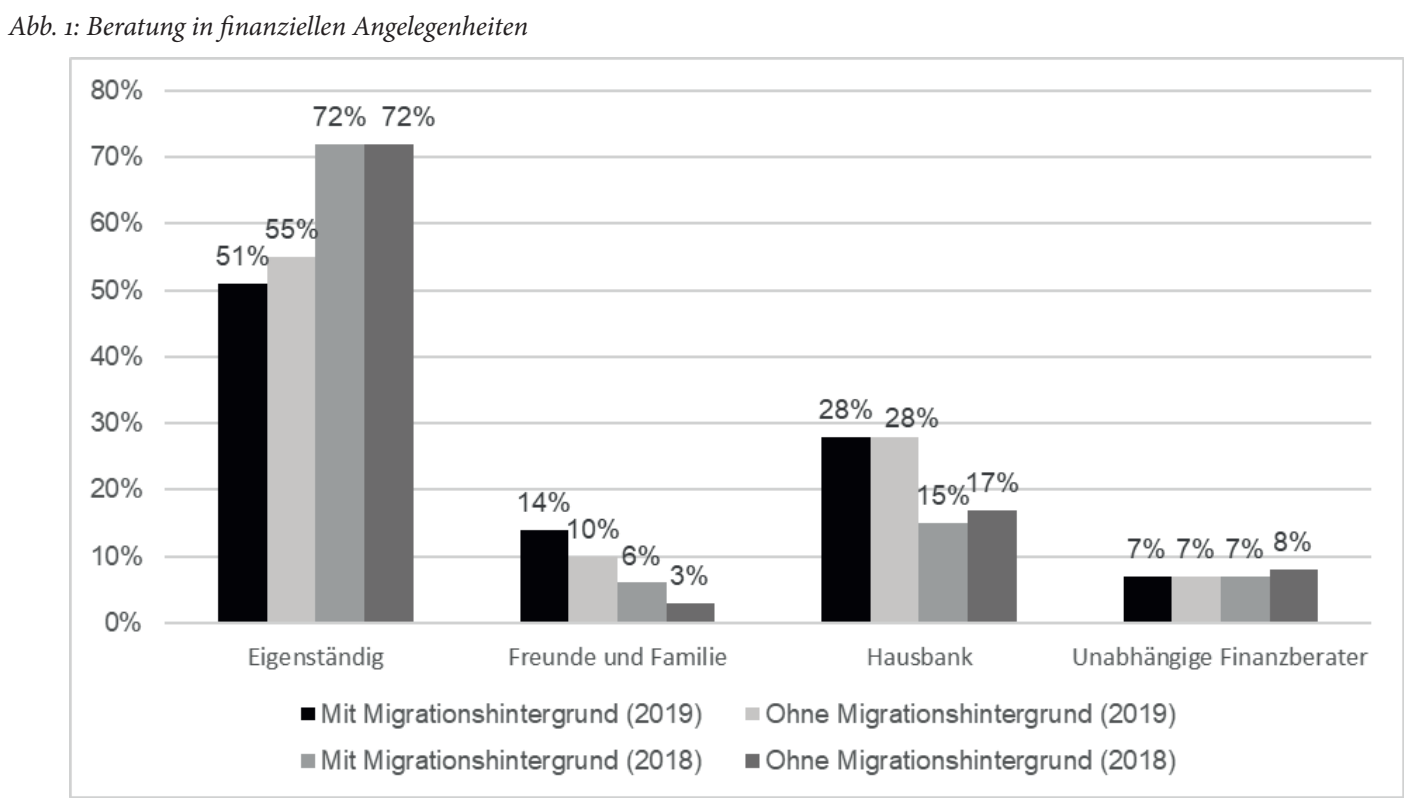

Quelle: Eigene Darstellung

Verständnis als „gut“ bis „sehr gut“ ein, wohingegen sich 65,88 Prozent der Befragten ohne Migrationshintergrund in diese Range einordnen.

Die folgenden Ergebnisse stimmen mit früheren Schlussfolgerungen überein, die keine signifikanten Unterschiede für Personen mit unterschiedlichen Ethnien aufweisen (de Bassa Scheresberg 2013; Lusardi/ Mitchell 2008). In der befragten Gruppe gibt es im Allgemeinen mehr Ähnlichkeiten als Unterschiede. Der analysierte Datensatz unterscheidet zwischen Forschungsteilnehmer und -teilnehmerinnen mit und ohne Migrationshintergrund. Es liegen keine weiteren Informationen zu den Befragten vor. Aus diesem Grund gibt es keine spezifischen Ergebnisse, z. B. für die religiösen, sozialen Rahmenbedingungen oder für das Heimatland der Menschen. Gleichzeitig gibt es keine gültigen Informationen über die Sprachkenntnisse der Befragten. Der Einfluss der jeweiligen Herkunftsländer wurde in dieser Empirie nicht analysiert. Im Folgenden werden vier Teile des Finanzverhaltens für Personen mit und ohne Migrationshintergrund analysiert:

Die Abbildung eins zeigt, dass es nur geringe Unterscheidungen zwischen den Befragten in den jeweiligen Betrachtungszeiträumen gibt. Personen ohne und mit Migrationshintergrund treffen ihre Entscheidungen im Kontext persönlicher Finanzthemen (51 bzw. 55 Prozent) meistens eigenständig. Falls dennoch jemand um Rat gebeten wird, ist der Berater oder die Beraterin der Hausbank die erste Anlaufstelle (28 Prozent). Im Vergleich zum Vorjahr ist somit ein Zuwachs von rund elf Prozent zu verzeichnen. Dieser Befund unterstreicht die Tatsache, dass die persönliche Beratung nach wie vor einen hohen Stellenwert bei den Privatkunden und Privatkundinnen genießt (Kahnhof/Zureck 2018). Da die eigenständigen Finanzentscheidungen innerhalb dieses einjährigen Zeitraums stark rückläufig sind, wird der Aspekt volatiler Märkte sowie steigender Risiken am Finanzmarkt unterstrichen (Deutsche Bundesbank 2020).

Im Kontext der Untersuchung des Investitionsverhaltens - „In welche Finanzprodukte investieren die Befragten?" -, sind vor allem zwei wesentliche Befunde hervorzuheben: Während Personen mit Migrationshintergrund neue digitale Investitionsmöglichkeiten in Form von Kryptowährungen deutlich offener gegenüberstehen (24 Prozent investieren in diese Assetklasse), ist das Anlageverhalten der Personen ohne Migrationshintergrund hierbei weniger stark ausgeprägt (lediglich 9 Prozent). Somit ist eine grundsätzlich höhere Offenheit zu innovativen Finanzprodukten ersichtlich. Ebenfalls ist zu betonen, dass Personen ohne Migrationshintergrund rund zehn Prozent häufiger in Kapitallebensversicherungen investieren. Somit lässt sich ein deutlich risikoaverseres Investitionsverhalten bei den Personen ohne Migrationshintergrund wahrnehmen (siehe hierzu Tabelle 1). Weil Befragte mit Migrationshintergrund verstärkt in risikoreichere Assetklassen investieren, können sie verstärkt der finanziellen Repression in Form der anhaltenden Niedrigzinsphase entgegenwirken. Im Gegensatz dazu droht bei Bevorzugung risikoarmer Investments ein langfristiger Renditeverzicht (Flossbach von Storch 2019). 
Tab. 1: Finanzprodukte

\begin{tabular}{lcccc}
\hline \multicolumn{1}{c}{ In welche Finanzprodukte wird investiert? } & Mit Migrationshintergrund (2019) & Ohne Migrationshintergrund (2019) \\
\hline Tagesgeldkonto & 26 & $51 \%$ & 203 & $66 \%$ \\
Termingeld/Festgeld/Sparbrief & 5 & $10 \%$ & 39 & $13 \%$ \\
Sparbuch & 17 & $33 \%$ & 122 & $39 \%$ \\
Bausparvertrag (ohne vermögenswirksame Leistungen) & 23 & $45 \%$ & 154 & $50 \%$ \\
Kapitallebensuersicherung & 9 & $18 \%$ & 83 & $27 \%$ \\
Aktien & 30 & $59 \%$ & 192 & $62 \%$ \\
Anleihen & 13 & $25 \%$ & 51 & $17 \%$ \\
Fonds & 26 & $51 \%$ & 191 & $62 \%$ \\
ETF (Exchange Traded Funds) & 24 & $47 \%$ & 126 & $41 \%$ \\
Zertifikate & 7 & $14 \%$ & 32 & $10 \%$ \\
Immobilien & 14 & $27 \%$ & 85 & $28 \%$ \\
Edelmetalle & 10 & $20 \%$ & 50 & $16 \%$ \\
Kryptowährungen & 12 & $24 \%$ & 27 & $9 \%$ \\
Anzahl der Befragten & & 51 & & 309 \\
\hline
\end{tabular}

Quelle: Eigene Darstellung

Abb. 2: Anlageziele 2018

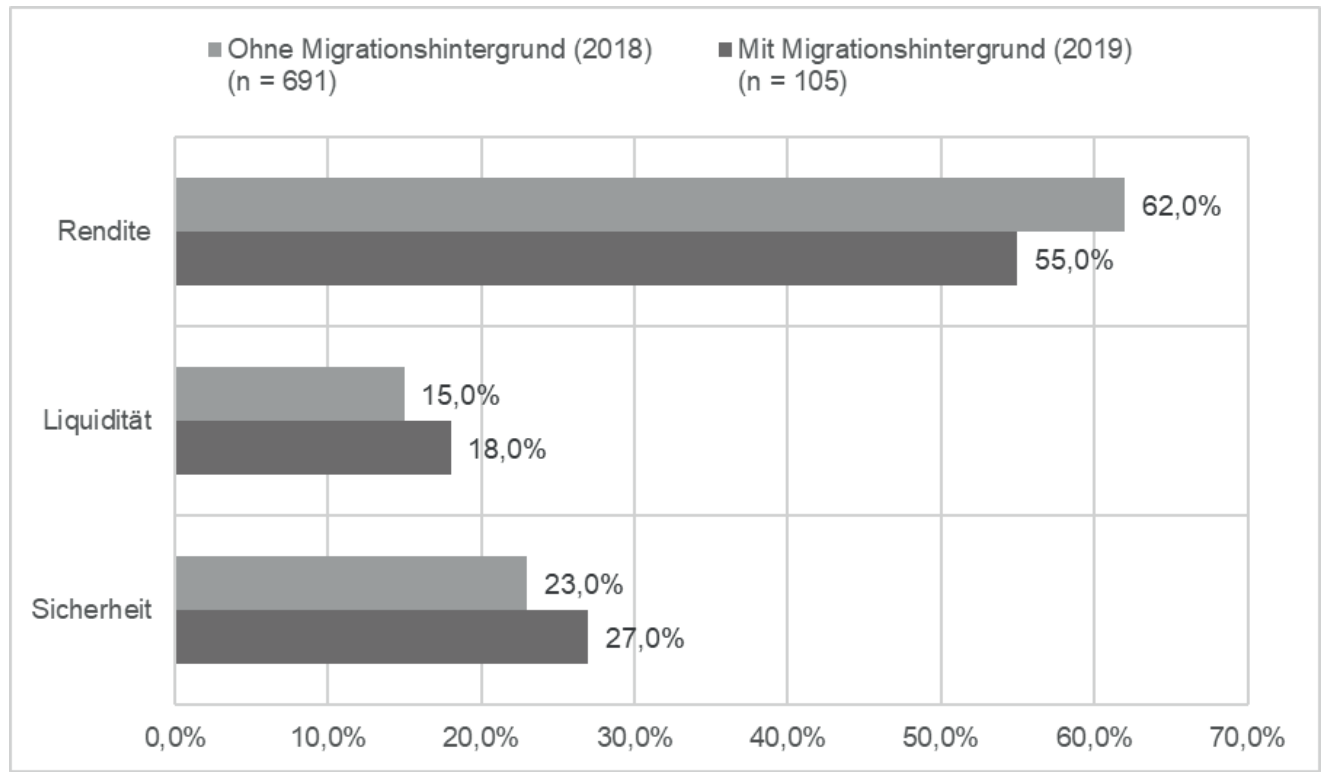

Quelle: Eigene Darstellung

In Deutschland konzentrieren sich die Anleger und Anlegerinnen im internationalen Vergleich nicht vorrangig auf die Rendite. In Bezug auf die Geschichte und die Gewohnheiten tendieren sie zu renditearmen Anlageformen mit besonderem Augenmerk auf Sicherheit (Lusardi/Mitchell 2011; Zureck et al. 2018). Die angesprochenen Gewohnheiten lassen sich vorrangig auf das deutsche Rentensystem zurückführen, da dieses bis in die letzten Jahrzehnte dem Großteil der
Bevölkerung die für den Ruhestand benötigten Renditen erwirtschaftet hat. Aufgrund der anhaltenden Niedrigzinsphase sind jedoch die Überschussrenditen von gesetzlichen und privaten Versicherungsinstitutionen stark rückläufig (Michels 2019). Wie Abbildung 4 zeigt, ist dieses Verhalten auch für Personen mit Migrationshintergrund in Deutschland im Befragungsjahr 2018 zutreffend. Während 2018 Verlustaversion, Sicherheit und Liquidität als Anlageziele für 


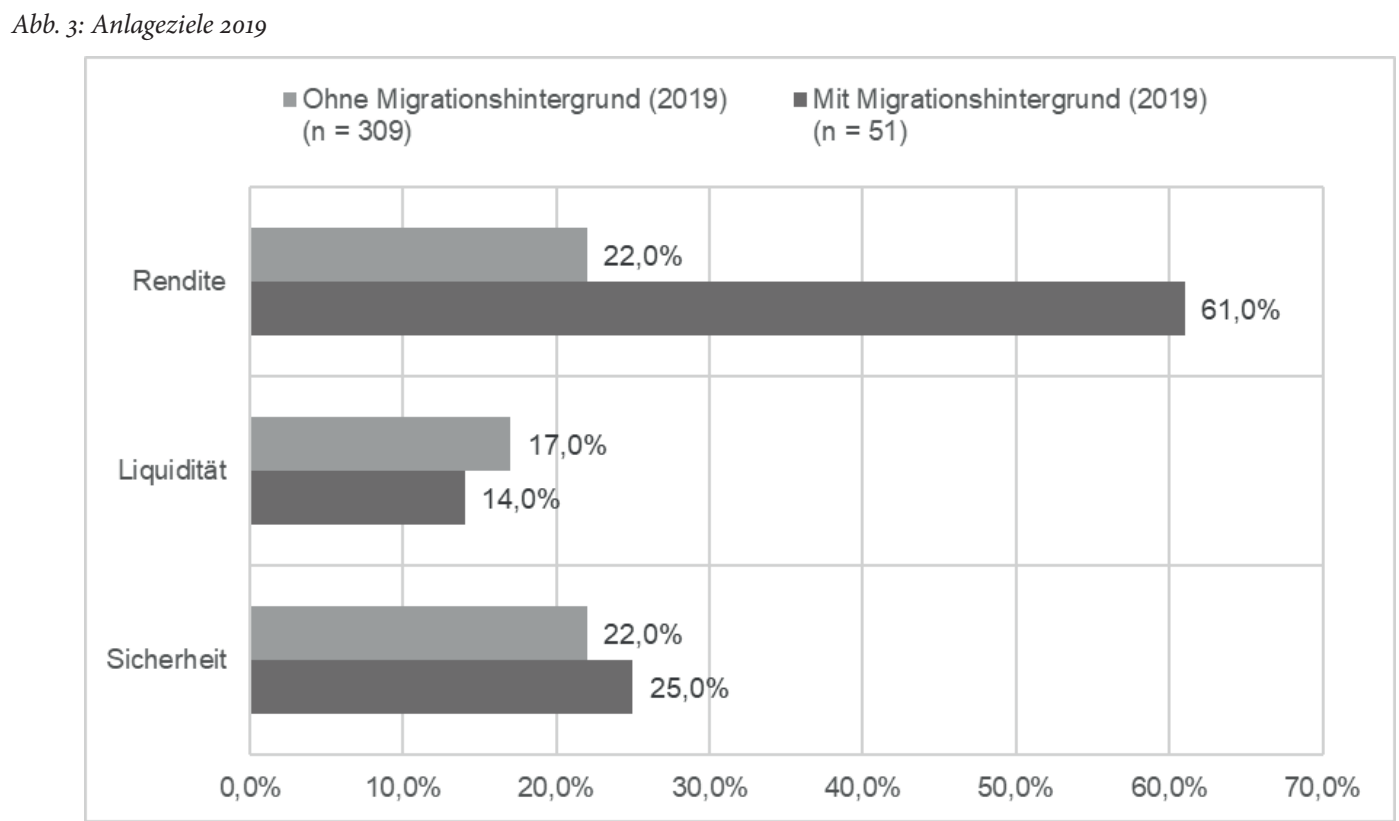

Quelle: Eigene Darstellung

Abb. 4: Wertentwicklung des Anlagebetrages in den vergangenen zwölf Monaten (Bezugsjahr 2018)

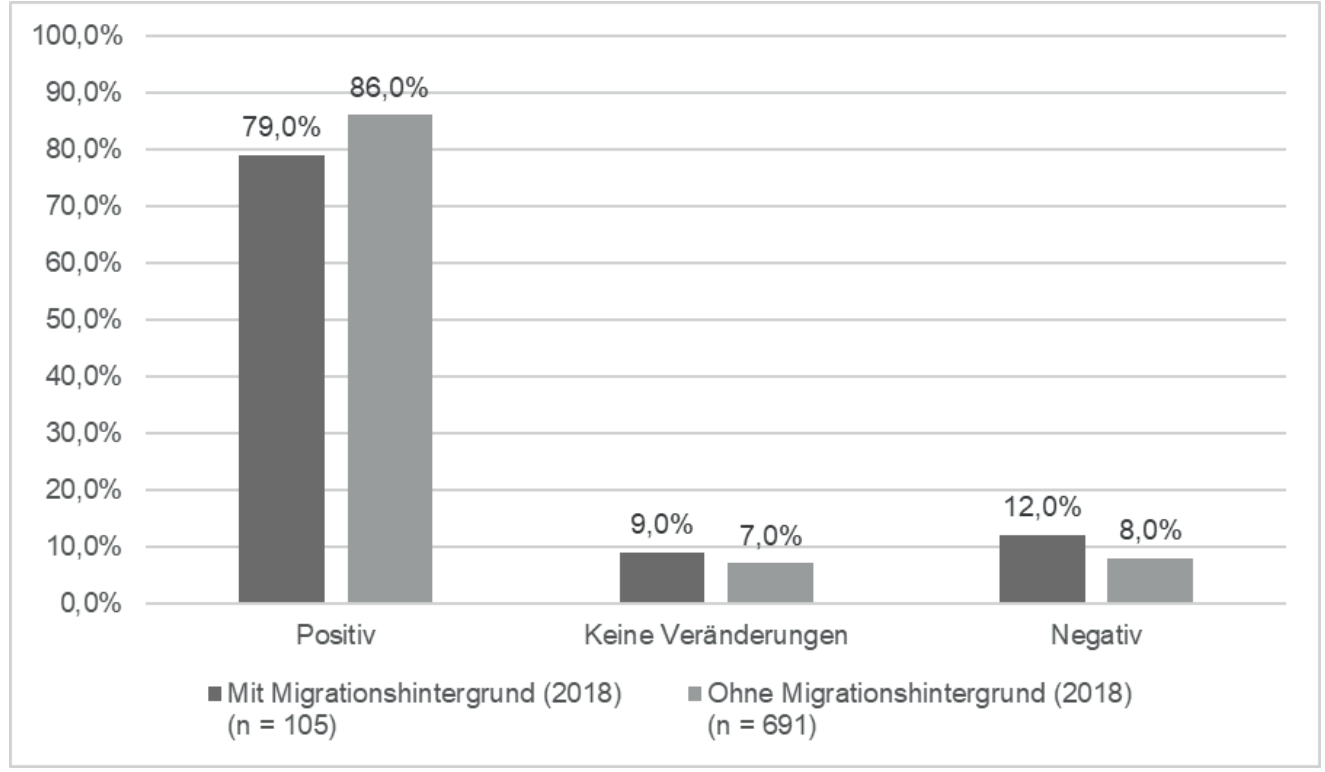

Quelle: Eigene Darstellung

Personen mit Migrationshintergrund wichtiger als für Personen ohne solchen waren, fokussiert sich 2019 die Mehrheit auf das Renditeziel. Dieses Investitionsziel ist folglich eine Strategie zur Vermeidung der finanziellen Repression. Dies lässt sich mit der stärker ausgeprägten Risikoneigung bei der Wahl der Investitionsgüter, siehe Abbildung 2 ebenfalls bestätigen.

Während 2018 Personen mit und ohne Migrationshintergrund nahezu die gleiche jährliche Performance mit ihren Investitionen erzielen, sind die Abweichungen 2019 höher. 82 Prozent der Personen ohne Migrationshintergrund erzielen eine positive Jahresperformance. Bei der anderen Gruppe sind dies jedoch nur 67 Prozent. Ebenfalls gibt es bei dem Eintrag in der Kategorie „Keine Veränderungen“ Abweichungen. In Bezug auf eine negative Jahresperformance befinden sich die Antworten beider Gruppen auf einem nahezu identischen Niveau, vergleiche hierzu Abbildungen 4 und 5. 


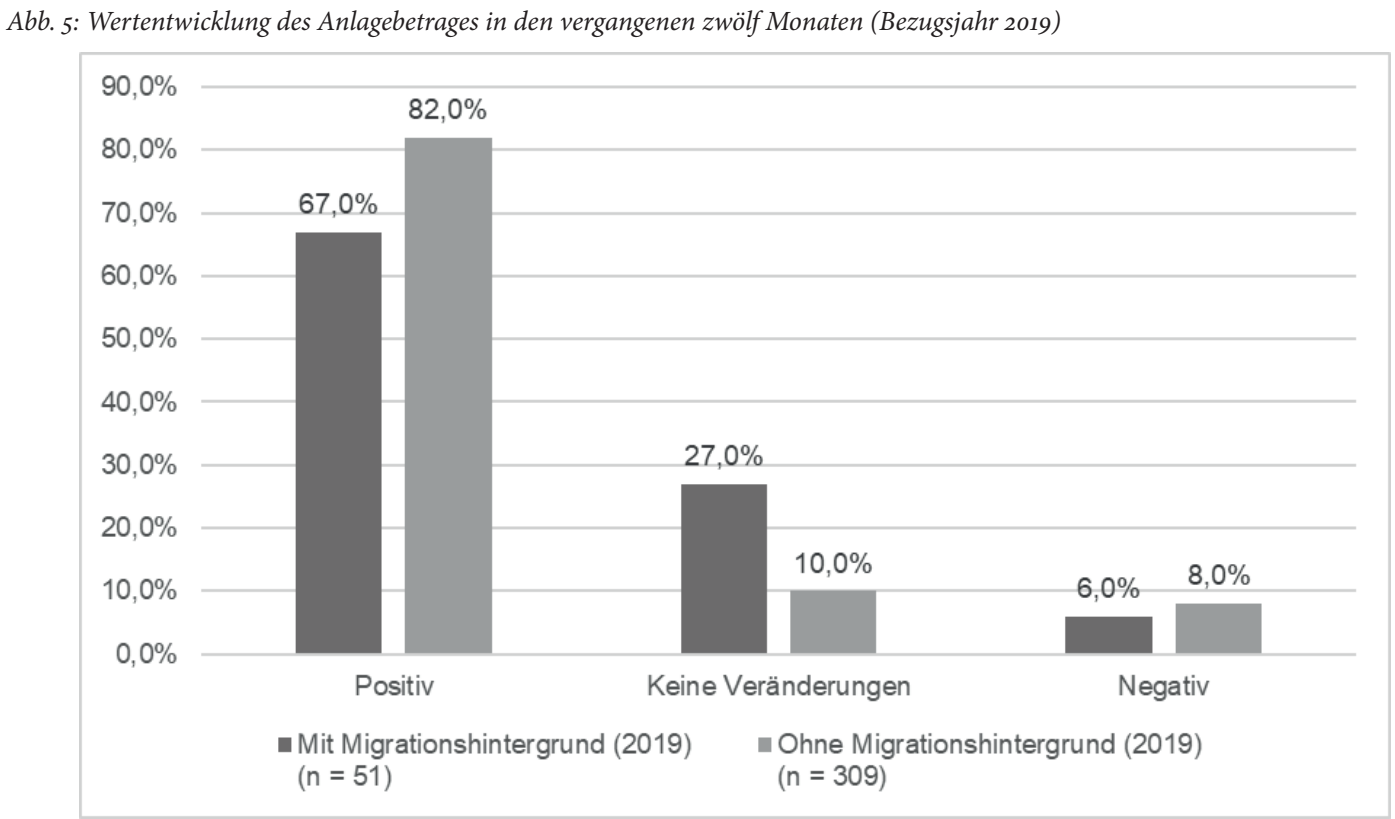

Quelle: Eigene Darstellung

Bei diesem deskriptiven Befund ist jedoch $\mathrm{zu}$ unterstreichen, dass dieser keinen direkten Zusammenhang zwischen der möglichen vorhandenen Financial Literacy sowie der Performance hervorhebt. Um diesen empirischen Zusammenhang zukünftig zu analysieren, wird versucht, die Anzahl der erhaltenen Antworten zu erhöhen. Ausgehend von einer größeren Datenmenge ist das Ziel die Durchführung einer logistischen Regression, um die Abhängigkeiten zwischen vorhandener Financial Literacy sowie der Performance der Befragten zu eruieren.

\section{Fazit und Ausblick}

Ziel des wissenschaftlichen Beitrages ist es, das Investitionsverhalten von in Deutschland lebenden Personen mit und ohne Migrationshintergrund $\mathrm{zu}$ analysieren. Daher konzentriert sich das Diskussionspapier auf die Finanzberatung, die selektierten Assets, die Anlageziele und die Wertentwicklung. Bei Beratungsbedarf bitten Personen mit und ohne Migrationshintergrund zunächst den Primärberater/die Primärberaterin in ihrer Hausbank um finanzielle Beratung. Familie und Freunde werden nicht so häufig um Rat gefragt. Es scheint, dass Geldangelegenheiten vertraulich sind und die Befragten im privaten Umfeld nicht darüber reden wollen. In beiden Gruppen dominieren eigenständige Finanzentscheidungen. Aufgrund der skizzierten Selbstentscheidung ist die politische Forcierung der all- gemeinen Finanzbildung in Deutschland stärker zu verfolgen (Institut der deutschen Wirtschaft 2019). Unter anderem durch verpflichtende Schulfächer könnte eine gute allgemeine Finanzbildung von Personen mit und ohne Migrationshintergrund in Deutschland sichergestellt werden. Ebenfalls ist zu unterstreichen, dass 2019 mehr Befragte auf ihre Hausbank zurückgreifen. Folglich ist die persönliche Beratung für Personen in Deutschland auch in Zeiten digitaler Veränderungen relevant (Krahnhof/Zureck 2018; Zureck/Krahnhof 2019).

Im Gegensatz zu Personen mit Migrationshintergrund achten Personen ohne Migrationshintergrund eher auf Sicherheit und Liquidität. In diesem Kontext ist jedoch $\mathrm{zu}$ betonen, dass ethische Investmentziele und -strategien, wie z. B. das Sharia Investing, nicht berücksichtigt wurden. Für diese Personen zählt es nicht so sehr, mit ihren Investitionen hohe Renditen $\mathrm{zu}$ erwirtschaften. Generell erzielen beide Beobachtungsgruppen mit ihren Anlagen überwiegend positive Renditen. Insgesamt gibt es keinen signifikanten Unterschied zwischen den Beobachtungsgruppen.

Festhalten lässt sich, dass die in der Empirie befragten Personen mit Migrationshintergrund häufiger in neue und somit auch risikoreichere Assetklassen, wie z. B. Kryptowährungen, investieren. Darüber hinaus unterscheiden sich die Anlageziele 2019 zwischen den beiden Gruppen. In der jüngsten Befragung legen vor allem Personen mit Migrationshintergrund darauf
MOMENTUM QUARTERLY IIIIIIIIIIIIIIIII 147 
Wert, Renditen zu erzielen. Insbesondere durch die Selektion von risikobehafteten Assets kann den individuellen Folgen der finanziellen Repression entgegengewirkt werden (Michels 2019). Durch entsprechend risikoreichere Assets können über einen längeren Betrachtungszeitraum höhere Renditen erwirtschaftet werden, die einen Bestandteil der privaten Altersvorsorge darstellen können. Diese spannende Entwicklung ist in den kommenden Jahren mit aktualisiertem Datenmaterial weiterzuverfolgen sowie durch weitere empirische Auswertungen zu vertiefen.

\section{Handlungsempfehlungen}

Da sich der Datensatz insbesondere auf Befragte im Alter zwischen 18 und 30 Jahren bezieht, konnten relevante Erkenntnisse zu dieser Altersgruppe gewonnen werden. Folglich beziehen sich die Forschungsergebnisse vorrangig auf „junge“ Personen. Die Studie betrachtet daher keine bzw. wenige Personen, die beispielsweise erst in einem höheren Lebensalter nach Deutschland kommen bzw. Personen mit Migrationshintergrund, die schon seit über 50 Jahren in Deutschland leben.

Der Beitrag verdeutlicht, dass Personen ohne Migrationshintergrund in Deutschland eher risikoavers agieren. Folglich werden renditearme Assetklassen, wie zum Beispiel Tages- und Sichteinlagen, langfristig renditestärkeren Assets gegenüber, wie zum Beispiel Wertpapiere oder Kryptowährungen, bevorzugt. Dieses Investitionsverhalten hat einen langfristigen Renditeverzicht zur Folge. Vor allem mit Blick auf die Auswirkungen der finanziellen Repression, basierend auf dem anhaltenden Niedrigzinsumfeld, kann es unter anderem zu einem individuellen Wohlstandsverlust im Rentenalter kommen, wie nachfolgende Daten unterstreichen.

Niedrige Geburtenraten und eine steigende Lebenserwartung haben in Deutschland $\mathrm{zu}$ einem stattfindenden demografischen Wandel geführt. Daher ist das Rentenniveau netto vor Steuern von 55,0 Prozent im Jahr 1990 auf 48,1 Prozent im Jahr 2018 gesunken (Bundesministerium für Arbeit und Soziales 2018). Unter anderem mit Blick auf das skizzierte sinkende Rentenniveau müssen Bürger und Bürgerinnen in Deutschland die private Altersvorsorge stärker verfolgen, um der Gefahr der Altersarmut proaktiv entgegenzuwirken. Um entsprechende Maßnahmen zur Risikoreduzierung der finanziellen Repression (z. B. teilweise Abkoppelung des Individuums vom wohlfahrtsstaatlichen System hin zur Ergreifung neoliberaler wirtschaftspolitischer Maßnahmen, wie zum Beispiel Aufbau einer privaten Altersvorsorge) $\mathrm{zu}$ ergreifen, bedarf es einer entsprechend positiven Financial Literacy.

Somit handelt es sich hierbei um ein praxisrelevantes Forschungsthema. Zwar ist die Erhöhung der allgemeinen Finanzbildung aufgrund des Föderalismus keine verpflichtende Aufgabe des Staates, nichtsdestotrotz sollte dieser durch entsprechende Förder- und Bildungsbeiträge aktiv zur Erhöhung beitragen und dadurch die Landespolitik ermutigen, sich der Thematik anzunehmen. Hierbei sollten Bund und Länder das differente (Investitions-)Verhalten von Personen mit und ohne Migrationshintergrund kritisch verfolgen, um zielgruppenspezifische Maßnahmen einzuleiten.

\section{Literatur}

Arthur, C. (2012): Financial Literacy Education Neoliberalism, the Consumer and the Citizen. Rotterdam/Boston: Sense publisher.

Belke, A./Keil, J. (2013): Niedrigzinsfalle: Die Gefahr der finanziellen Repression. Vierteljahrshefte zur Wirtschaftsforschung, 82 (2), 113-125.

Bundesministerium für Arbeit und Soziales (2019): Stichwort: Rentenniveau. Online: https://www.bmas.de/ $\mathrm{DE} /$ Themen/Rente/Rentenlexikon/Functions/glossar. html?cms_lv2 $=753068 \mathrm{cms} \_l v 3=86064$ \#glossar86064 [29.03.2020]

Black, S. E./Devereux, P. J./Lundborg, P./Majlesi, K (2017): On the Origins of Risk-Taking in Financial Markets. Journal of Finance, 72 (5), 2229-2278.

De Bassa Scheresberg, C. (2013): Financial Literacy and Financial Behavior among Young Adults: Evidence and Implications. Numeracy, 6 (2), Article 5.

Destatis Statistisches Bundesamt (2019): Jede vierte Person in Deutschland hatte 2018 einen Migrationshintergrund. Pressemitteilung Nr. 314 vom 21. August 2019. Online: https://www.destatis.de/DE/Presse/Pressemitteilungen/2019/08/PD19_314_12511.html [14.08.2020].

Deutsches Aktieninstitut (2019): Aktionärszahlen des deutschen Aktieninstituts 2018. Online: https://www. dai.de/files/dai_usercontent/dokumente/studien/2019-03-06\%20Aktieninstitut\%20Aktionaerszahlen\%202018.pdf [14.08.2020].

Deutsche Bundesbank (2020): Monatsbericht Mai 2020. Online: https://www.bundesbank.de/resource/blob/832766 /25cd 9849 e 74 fio2d 4794382230 ao $96 \mathrm{ag} / \mathrm{mL} / 2020-05-\mathrm{mo}-$ natsbericht-data.pdf [14.08.2020].

Deutsches Institut für Altersvorsorge (2019): Finanzbildung mit Fundament: Fehlanzeige. Aufwelchen Wegen gelangen 
Jugendliche zu mehr Finanzwissen. Online: https://www. dia-vorsorge.de/wp-content/uploads/2019/12/DIA-Studie-Finanzbildung_05.12.2019_ES.pdf [14.08.2020].

Deutsche Rentenversicherung Bund (2019): Stichwort: Rentenniveau: Wie weit kann das Rentenniveau bis 2030 sinken? Online: https://www.deutsche-rentenversicherung.de/DRV/DE/Rente/Allgemeine-Informationen/ Wissenswertes-zur-Rente/FAQs/Rente/Rentenniveau/ Rentenniveau_Liste.html\#ad901b16-b989-47af-847e$52 \mathrm{aff}_{3} \mathrm{~d}_{20074}[02.09 .2020]$.

Ferguson, Roger W. (2002): Reflections on Financial Literacy. Remarks by Vice Chairman Roger W. Ferguson, Jr. Before the National Council on Economic Education. Online: www.federalreserve.gov/boarddocs/speeches/2002/20020513/default.htm [13.07.2020.

Financial Literacy \& Education Russia Trust Fund (2013): Improving financial education effectiveness through behavioural economics. Online: https://www.oecd.org/daf/ fin/financial-education/TrustFund2013_OECDImproving_Fin_Ed_effectiveness_through_Behavioural_Economics.pdf [14.08.2020].

Flossbach von Storch (2019): Seit acht Jahren verlieren Sparer regelmäßig Geld. Online: https://www.private-bankingmagazin.de/flossbach-von-storch-seit-acht-jahren-verlieren-sparer-regelmaessig-geld-1/ [13.07. 2020].

Haan, P./Stichnoth, H./Blömer, M./Buslei, H./Geyer, J./Krolage, C./Müller, K.-U. (2017): Entwicklung der Altersarmut bis 2036: Trends, Risikogruppen und Politikszenarien. Gütersloh: Bertelsmann Stiftung.

Haliassos, M./Jansson, T./Karabulut, Y. (2017): Incompatible European Partners? Cultural Predispositions and Household Financial Behavior. Management Science, 63 (11), 3780-38o8.

Hastings, J. S./Madrian, B. C./Skimmyhorn, W. L. (2013): Financial Literacy, Financial Education, and Economic Outcomes. Annual Review of Economics, 5 (1), 347-373.

Homburg, S./Herz, B./Erler, A./Mayer, T./Heise, A./Neyer, U. (2013): Finanzielle Repression - ein Instrument zur Bewältigung der Krisenfolgen? Wirtschaftsdienst, 93 (11), 731-750.

Hung, A./Parker, A. M./Yoong, J. (2009): Defining and measuring financial literacy. Rand Working Paper. Online: https://www.rand.org/content/dam/rand/pubs/working papers/2009/RAND_WR708.pdf [02.09.2020].

Institut der deutschen Wirtschaft (2019): INSM-Bildungsmonitor 2019 - Schwerpunktthema „Ökonomische Bildung": Gutachten für die Initiative Neue Soziale Marktwirtschaft. Online: https://www.insm.de/fileadmin/insm-dms/ downloads/INSM-Bildungsmonitor-Schwerpunktthema-2019.pdf [14.08.2020].

Institut der Wirtschaftsprüfer (2019): Zunahme der Staatsverschuldung und mögliche Auswirkungen auf den Finanzsektor. Online: https://www.idw.de/blob/120686/1a4c8 8d53c14C7298f2ea194ec978a79/down-positionspapierstaatsverschuldung-data.pdf [14.08.2020].

Michels, J. (2019): Gewinner und Verlierer in der Welt der dauerhaften Niedrigzinsen. Online: https://www.
ifo.de/DocDL/sd-2019-20-michels-etal-niedrigzinsen-2019-10-24.pdf [14.08.2020].

Krahnhof, P./Zureck, A. (2018): Konsequenzen aus Digitalisierung und Konsolidierung für kleinere Institute. Zeitschrift für das gesamte Kreditwesen, 18, 922.

Köhler, Z. (2017): ING-DiBa-Studie: Hälfte der Deutschen sind finanzielle Analphabeten: Deutschland, im europäischen Vergleich nur Vorletzter. Online: https://www.ing.de/ ueber-uns/presse/pressemitteilungen/finanzielle-analphabeten/ [14.08.2020].

Lusardi, A./Mitchell, O. S. (2011): Financial Literacy around the world: an overview. Journal of Pension Economics \& Finance, 10 (4), 497-508.

Lusardi, A./Mitchell, O. S./Curto, V. (2010): Financial Literacy among the Young. Journal of Consumer Affairs, 44 (2), 358-380.

Mayer, M. (2015): Zuwanderungsbedarf aus Drittstaaten in Deutschland bis 2050: Szenarien für ein konstantes Erwerbspotenzial - unter Berücksichtigung der zukünftigen inländischen Erwerbsbeteiligung und der EU-Binnenmobilität. Gütersloh: Bertelsmann Stiftung.

McKinnon, Ronald I. (1973): Money and Capital in Economic Development. Washington, D. C.: Brookings Institute.

Schneider-Reißig, M. (2018): Debt Literacy. Konzeption und Messansatz zum Kreditwesen des Verbrauchers. Wiesbaden: Springer, 7-49.

Shaw, E. S. (1973): Financial Deepening in Economic Development. NewYork: Oxford University Press.

Vargas, M. (2012): Bedeutung der finanziellen Entwicklung im Aufholprozess von Entwicklungs- und Schwellenländern: Eine vergleichende Analyse der chilenischen und mexikanischen Erfahrungen. Stuttgart: Lucius \& Lucius Verlag.

Özcan, U./Lehrbass, F./Zureck, A. (2018): Investieren Migranten anders? Die Bank, 6, 22-25.

YourHub (2019): Forming Habits at a Young Age: Safeway Commits to Developing Youth Financial Education. Online: https://yourhub.denverpost.com/blog/2019/o3/ safeway-commits-to-developing-youth-financial-education/238819/ [03.02.2020].

Zimmermann, G./Baier, F. (2012): Financial Repression - Ein neues Umfeld für die Finanzmärkte? Wirtschaftsdienst, 92 (9), 599-604.

Zureck, A./Jäger, T. (2018): Migration Background and its Impact on Personal Investments of in Germany Living People. International Journal of Innovation and Economic Development, 4 (3), 7-11.

Zureck, A./Krahnhof, P. (2019): Der richtige Umgang mit Digitalisierung. Bank intern, 22 (9), 1-2.

Zureck, A./Krahnhof, P. (2017): Digitalisierung und Auswirkungen auf das Retail-Banking von morgen. $b d v b$ aktuell, 137, 28-29.

Zureck, A./Reiter, J./Svoboda, M. (2018): Cross-Generational Investment Behavior and the Impact on Personal Finance. Journal of International Business Research and Marketing, 3 (2), 16-18. 
Zureck, A./Svoboda, M. (2015): Kritische Analyse der Financial Literacy im internationalen Vergleich. In: Frère, E./ Reuse, S. (Hg.): 7. Forschungsforum Finance: Risk- und Investmanagement am Finanz- und Kapitalmarkt. Essen: MA Akademie Verlags- und Druck-Gesellschaft, 19-31. 